\title{
Synthesis of Spatial RPRP Loops for a Given Screw System
}

\author{
A. Perez-Gracia \\ Institut de Robotica i Informatica Industrial (IRI) UPC/CSIC, Barcelona, Spain \\ and: College of Engineering, Idaho State Univesity, USA \\ e-mail:aperez@iri.upc.edu
}

\begin{abstract}
The dimensional synthesis of spatial chains for a prescribed set of positions can be used for the design of parallel robots by joining the solutions of each serial chain at the end effector. In some cases, this may yield a system with negative mobility. The synthesis of some overconstrained but movable linkages can be done if the finite screw system associated to the motion of the linkage is known. For these cases, the screw system could be related to the finite tasks positions traditionally defined in the synthesis theory. This paper presents the simplest case, that of the spatial RPRP closed chain, for which one solution exists.
\end{abstract}

Key words: Dimensional synthesis, overconstrained linkages, finite screw systems.

\section{Introduction}

Synthesis of parallel robots has focused mainly on type or structural synthesis, using group theory, screw theory, or geometric methods, see for instance [3]. Dimensional synthesis examples exist, which focus on optimizing performance indices [7], [10] or on reachable workspace sizing [12], [2]; see also [14].

The dimensional synthesis of spatial serial chains for a prescribed set of positions can be used for the design of parallel robots by synthesizing all supporting legs for the same set of positions. There are a few examples of finite-position dimensional synthesis of parallel robots in the literature, most of them doing partial synthesis. Wolbrecht et al. [21] perform synthesis of 3-RRS, 4-RRS and 5-RRS symmetric parallel manipulators; Kim and Tsai [11] and Rao [20] solve the partial kinematic synthesis of a 3-RPS parallel manipulator. This method yields, in some cases, a system with negative mobility.

One interesting question is whether the finite-screw surfaces generated by the task positions can give any information for the synthesis of the overconstrained closed linkages. Using Parkin's definition for pitch [15], the screws corresponding to finite displacements can form screw systems. Huang [4] showed that the single RR chain forms a finite screw system of third order; however, the set of finite displacements of the coupler of the Bennett linkage form a cylindroid, which is a 
general 2-system of screws [5]. Baker [1] has also studied the motion of the Bennett linkage. Perez and McCarthy [16] used two arbitrary displacements to generate the cylindroid associated to the Bennett linkage in order to perform dimensional synthesis. Husty et al. [9] use the geometry of the Study quadric to obtain simpler equations for the synthesis and analysis. Following this approach, Pfurner and Husty [19] present the constraint manifold of overconstrained 2-3R parallel robots as $6 \mathrm{R}$ closed chains.

In this paper, the focus is on the simplest of the overconstrained linkages, the closed spatial RPRP linkage. Recently, Huang [6] has shown that the set of screws corresponding to displacements of this linkage forms a 2-screw system. We use this result in order to synthesize RPRP linkages with positive mobility and for a given shape of the screw system of the relative displacements. In order to do so, we state the design equations using the Clifford algebra of dual quaternions [18], which has a direct relation to the screw system. The design yields a single RPRP linkage.

\section{Clifford algebra equations for the synthesis}

\subsection{Forward Kinematics}

The approach used in this paper for stating design equations is based on the method of Lee and Mavroidis [13]. They equate the forward kinematics of a serial chain to a set of goal displacements and consider the Denavit-Hartenberg parameters as variables. A more efficient formulation for our purpose consists of stating the forward kinematics of relative displacements using the even Clifford subalgebra $C^{+}\left(P^{3}\right)$, also known as dual quaternions. In this section, we follow the approach presented in [18].

The Plücker coordinates $S=(\mathbf{s}, \mathbf{c} \times \mathbf{s})$ of a line can be identified with the Clifford algebra element $\mathbf{S}=\mathbf{s}+\varepsilon \mathbf{c} \times \mathbf{s}$. Similarly, the screw $\mathrm{J}=(\mathbf{s}, \mathbf{v})$ becomes the element $J=\mathbf{s}+\varepsilon \mathbf{v}$. Using the Clifford product we can compute the exponential of the screw $\frac{\theta}{2} \mathrm{~J}$,

$$
e^{\frac{\theta}{2} J}=\left(\cos \frac{\theta}{2}-\frac{d}{2} \sin \frac{\theta}{2} \varepsilon\right)+\left(\sin \frac{\theta}{2}+\frac{d}{2} \cos \frac{\theta}{2} \varepsilon\right) S=\cos \frac{\hat{\theta}}{2}+\sin \frac{\hat{\theta}}{2} S .
$$

The exponential of a screw defines a unit dual quaternion, which can be identified with a relative displacement from an initial position to a final position in terms of a rotation around and slide along an axis.

For a serial chain with $n$ joints, in which each joint can rotate an angle $\theta_{i}$ around, and slide the distance $d_{i}$ along, the axis $\mathrm{S}_{i}$, for $i=1, \ldots, n$, the forward kinematics of relative displacements (with respect to a reference position) can be expressed as the composition of Clifford algebra elements. Let $\theta_{0}$ and $\mathbf{d}_{0}$ be the joint parameters of this chain when in the reference configuration, so we have $\Delta \hat{\theta}=\left(\theta-\theta_{0}+(\mathbf{d}-\right.$ $\left.\mathbf{d}_{\mathbf{0}}\right) \varepsilon$ ). Then, the movement from this reference configuration is defined by 


$$
\begin{aligned}
\hat{Q}(\Delta \hat{\theta}) & =e^{\frac{\Delta \hat{\theta}_{1}}{2}} \mathrm{~S}_{1} e^{\frac{\Delta \hat{\theta}_{2}}{2}} \mathrm{~S}_{2} \ldots e^{\frac{\Delta \hat{\theta}_{n}}{2} \mathrm{~S}_{n}}, \\
& =\left(\mathrm{c} \frac{\Delta \hat{\theta}_{1}}{2}+\mathrm{s} \frac{\Delta \hat{\theta}_{1}}{2} \mathrm{~S}_{1}\right)\left(\mathrm{c} \frac{\Delta \hat{\theta}_{2}}{2}+\mathrm{s} \frac{\Delta \hat{\theta}_{2}}{2} \mathrm{~S}_{2}\right) \cdots\left(\mathrm{c} \frac{\Delta \hat{\theta}_{n}}{2}+\mathrm{s} \frac{\Delta \hat{\theta}_{n}}{2} \mathrm{~S}_{n}\right) .
\end{aligned}
$$

Note that $\mathrm{s}$ and $\mathrm{c}$ denote the sine and cosine functions, respectively.
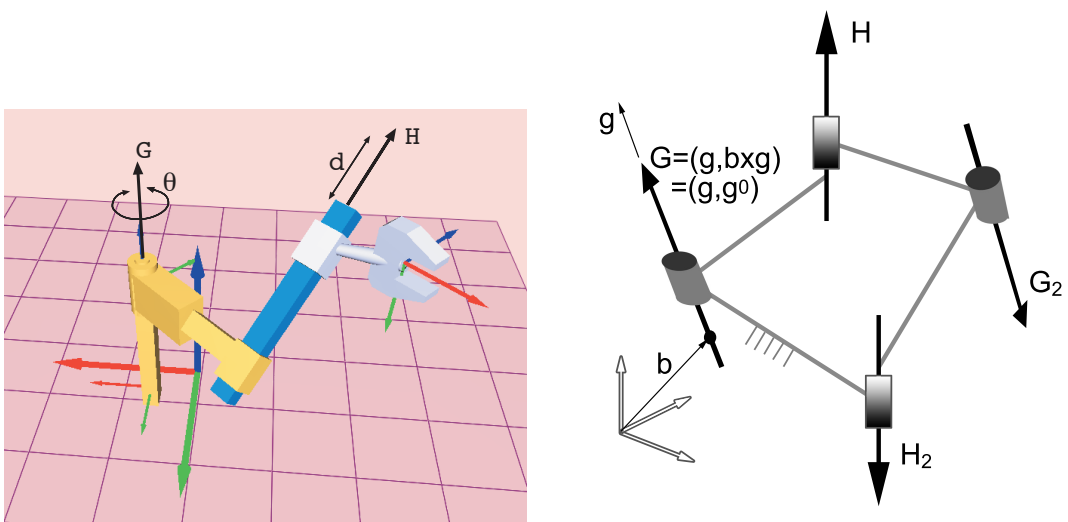

Fig. 1 The RP serial chain, left; the RPRP closed linkage, right.

The RPRP linkage has a mobility $M=-2$ using the Kutzbach-Groebler formula; however, for certain dimensions of the links, it moves with one degree of freedom. The RPRP linkage can be seen as a serial RP chain and a serial PR chain joined at their end-effectors.

The RP serial chain consists of a revolute joint followed by a prismatic joint. Figure 1 shows the RP serial chain and a sketch of the RPRP linkage with its axes. In the PR serial chain, the order of the joints in the chain is switched. For both the $\mathrm{RP}$ and PR serial chains, let $\mathrm{G}=\mathbf{g}+\varepsilon \mathbf{g}^{0}$ be the revolute joint axis, with rotation $\theta$, and $\mathbf{H}=\mathbf{h}+\varepsilon \mathbf{h}^{0}$ the prismatic joint axis, with slide $d$. Notice that, for synthesis purposes, the location of the slider, given by $\mathbf{h}^{0}$, is irrelevant. The Clifford algebra forward kinematics equations of the RP chain are

$$
\begin{aligned}
& \hat{Q}_{R P}(\Delta \theta, \Delta d)=\left(\cos \frac{\Delta \theta}{2}+\sin \frac{\Delta \theta}{2} \mathrm{G}\right)\left(1+\varepsilon \frac{\Delta d}{2} \mathrm{H}\right)= \\
& \left(\mathrm{c} \frac{\Delta \theta}{2}+\mathrm{s} \frac{\Delta \theta}{2} \mathbf{g}\right)+\varepsilon\left(-\frac{\Delta d}{2} \mathrm{~s} \frac{\Delta \theta}{2} \mathbf{g} \cdot \mathbf{h}+\frac{\Delta d}{2} \mathrm{c} \frac{\Delta \theta}{2} \mathbf{h}+\mathrm{s} \frac{\Delta \theta}{2} \mathbf{g}^{0}+\frac{\Delta d}{2} \mathrm{~s} \frac{\Delta \theta}{2} \mathbf{g} \times \mathbf{h}\right) .
\end{aligned}
$$

For the $P R$ chain, the only difference is a negative sign in the cross product. 


\subsection{Design Equations and Counting}

The design equations are created when a set of task positions are defined. The design variables that determine the dimensions of the chain are the position of the joint axes in the reference configuration.

Given a set of task positions expressed as relative displacements, $\hat{P}_{1 j}=\cos \frac{\Delta \hat{\phi}_{1 j}}{2}+$ $\sin \frac{\Delta \hat{\phi}_{1 j}}{2} \mathrm{P}_{1 j}, j=2, \ldots, m$, we equate them to the forward kinematics in Eq. (2),

$$
\hat{P}_{1 j}=e^{\frac{\Delta \hat{\theta}_{1 j}}{2} \mathrm{~S}_{1}} e^{\frac{\Delta \hat{\theta}_{2 j}}{2} \mathrm{~S}_{2}} \cdots e^{\frac{\Delta \hat{\theta}_{n j}}{2} \mathrm{~S}_{n}}, \quad j=2, \ldots, m
$$

The result is $8(m-1)$ design equations. The unknowns are the $n$ joint axes $\mathrm{S}_{i}, i=$ $1, \ldots, n$, and the $n(m-1)$ pairs of joint parameters $\Delta \hat{\theta}_{i j}=\Delta \theta_{i j}+\Delta d_{i j} \varepsilon$.

For the RP (and similarly the PR) serial chains, the design equations are

$$
\hat{Q}_{R P}\left(\Delta \theta^{j}, \Delta d^{j}\right)=\hat{P}_{1 j}, \quad j=1, \ldots, m .
$$

The counting of independent equations and unknowns allows to define the maximum number of arbitrary positions $m$ that can be reached, based only on the type and number of joints of the serial chain, see [17] for details. Consider a serial chain with $r$ revolute and $p$ prismatic joints. The maximum number of task positions is given by $m$ in Eq.(6).

For serial chains with less than three revolute joints, the structure of semi-direct product of the composition of displacements needs to be considered, and the maximum number of rotations $m_{R}$ needs to be calculated too. Assuming that the orientations are given and that both the directions of the revolute joints and the angles to reach the task orientations are known, we can count, in a similar fashion, the number of translations $m_{T}$ that the chain can be defined for,

$$
m=\frac{3 r+p+6}{6-(r+p)}, \quad m_{R}=\frac{3+r}{3-r}, \quad m_{T}=\frac{2 r+p+3-c}{3-p} .
$$

In order to determine the maximum number of task positions for the RP and PR chains, we apply Eq. (6), to obtain $m=2.5$ task positions. Additional information is obtaining when computing $m_{R}=2$ task rotations, and $m_{T}=3$ task translations. Hence, we can define one arbitrary relative displacement and a second relative displacement whose orientation is not general.

\section{Screw system for the RPRP Linkage}

In the context of this paper, a screw surface is a ruled surface in which the lines correspond to relative displacements. A screw surface will be a screw system if it 
is closed under addition and scalar multiplication, that is, if it can be written as a linear combination of screws.

The linear combination of two arbitrary screws representing relative displacements form a 2-system known as the cylindroid, which is the manifold for the relative displacements of the closed 4R linkage. Huang [6], by intersecting the 3systems associated with the RP and PR dyads, shows that the screw surface of the closed RPRP linkage forms a 2-system of a special type, the fourth special type according to Hunt [8], also known as 2-IB [22]. The screws of this system are parallel, coplanar screws whose pitches vary linearly with their distance.

This screw system can be generated by two screws with same direction and finite pitches. Notice that this coincides with the results of the counting for the synthesis of the RP (or PR) serial chain. This allows us to define and use the screw system as input for the dimensional synthesis of the closed RPRP chain.

We have several strategies for doing so. For instance, we can select a first relative displacement, $\hat{S}_{12}=\cos \frac{\hat{\Delta \psi}}{2}+\sin \frac{\hat{\Delta}}{2}\left(\mathbf{s}_{12}+\varepsilon \mathbf{s}_{12}^{0}\right)$. The rotation axis of the displacement, $\mathbf{s}_{12}$ is common to both $\hat{S}_{12}$ and the second relative displacement. We set $\mathbf{s}_{12}=\mathbf{s}_{13}$ and select a rotation angle to define the relative rotation $\hat{s}_{13}$.

We can then set the slope of the pitch distribution in order to shape the screw system. The pitch for the finite displacement screws is [15]

$$
p_{1 i}=\frac{\frac{\Delta t_{1 i}}{2}}{\tan \frac{\Delta \psi_{1 i}}{2}}
$$

directly calculated from the dual quaternion using $p_{1 i}=\frac{\mathbf{s}_{1 i} \cdot \mathbf{S}_{i i}^{0}}{\mathbf{S}_{1 i} \cdot \mathbf{S}_{1 i}}$. Similarly, a point on the screw axis is calculated as

$$
\mathbf{c}_{1 i}=\mathbf{s}_{1 i} \times \mathbf{s}_{1 i}^{0}
$$

Define the slope of the distribution as

$$
K=\frac{p_{13}-p_{12}}{\left\|\mathbf{c}_{13}-\mathbf{c}_{12}\right\|}
$$

If we set the value of $K$, we can solve for $\Delta t_{13}$ in order to define the pitch of the second relative displacement, the location of its screw axis being defined. This defines the screw system; by converting to absolute displacements, we can easily check whether the trajectory of the end-effector is acceptable.

\section{Dimensional Synthesis of the RPRP Linkage for a Prescribed Screw System}

The solution of the RP, and similarly, PR chains is simple and yields one solution. Given an arbitrary relative displacement $\hat{Q}_{12}=\left(q_{12}^{w}+\mathbf{q}_{12}\right)+\varepsilon\left(q_{12}^{w 0}+\mathbf{q}_{12}^{0}\right)$ and a sec- 
ond displacement $\hat{Q}_{13}$ such that both have same direction and a given pitch distribution, as explained in previous section, we equate them to the forward kinematics in Eq.(3). We can solve for the direction of the revolute joint $\mathbf{g}$ and the rotation angles,

$$
\mathbf{g}=\frac{\mathbf{q}_{12}}{\left\|\mathbf{q}_{12}\right\|}, \quad \tan \frac{\Delta \theta_{1 i}}{2}=\frac{\left\|\mathbf{q}_{1 i}\right\|}{q_{1 i}^{w}}, \quad i=2,3 .
$$

The equations for the dual part are linear in the moment of the revolute joint, $\mathbf{g}^{0}$,

$$
\mathbf{g}^{0}=\frac{1}{\sin \frac{\Delta \theta_{1 i}}{2}}\left(\mathbf{q}_{1 i}^{0}-\frac{\Delta d_{1 i}}{2}\left(\cos \frac{\Delta \theta_{1 i}}{2} \mathbf{h}+\sin \frac{\Delta \theta_{1 i}}{2} \mathbf{g} \times \mathbf{h}\right)\right), \quad i=1,2
$$

Equating the solution of $\mathbf{g}^{0}$ for both relative displacements, we can solve linearly for $\mathbf{h}$ as a function of the slides $\Delta d_{12}, \Delta d_{13}$. The relation between the slides is given by the pitch condition,

$$
\frac{q_{12}^{w 0}}{\frac{\Delta d_{12}}{2} \sin \frac{\Delta \theta_{12}}{2}}=\frac{q_{13}^{w 0}}{\frac{\Delta d_{13}}{2} \sin \frac{\Delta \theta_{13}}{2}}
$$

Imposing $\|h\|=1$, we can solve for the slides to obtain one solution.

Using the same process, we can solve for the PR serial chain.

\section{Example}

The dual quaternions in Table 1 were generated as explained. $\hat{S}_{12}$ has been randomly generated, while the rotation in $\hat{S}_{13}$ is such that it belongs to the workspace of the chain. We select $\mathbf{c}_{13}=(-1.237,2.541,-1.601)$, randomly generated. Set a value of the slope of the pitch distribution, $K=0.48$, to create the second displacement.

Table 1 Goal relative displacements for the RP and PR chains

$$
\begin{aligned}
& (0.459,-0.133,-0.565,-0.672)+\varepsilon(1.660,0.343,-0.019,1.082) \\
& (0.135,-0.039,-0.167,0.976)+\varepsilon(0.023,-0.570,-0.923,-0.184)
\end{aligned}
$$

These two screws generate the screw system in Figure 2, where the length of each screw is proportional to its pitch. Some of the corresponding absolute displacements of the trajectory are included in the same Figure.

We obtain one solution for the RPRP linkage, specified in Table 2 as the Plücker coordinates of the axes and the joint variables to reach the positions.

Comparing these results to the joint variable conditions in [6] we can see that our solution corresponds to the unfolded RPRP linkage. Figure 3 shows the chain reaching the three displacements, using the identity as reference displacement. 

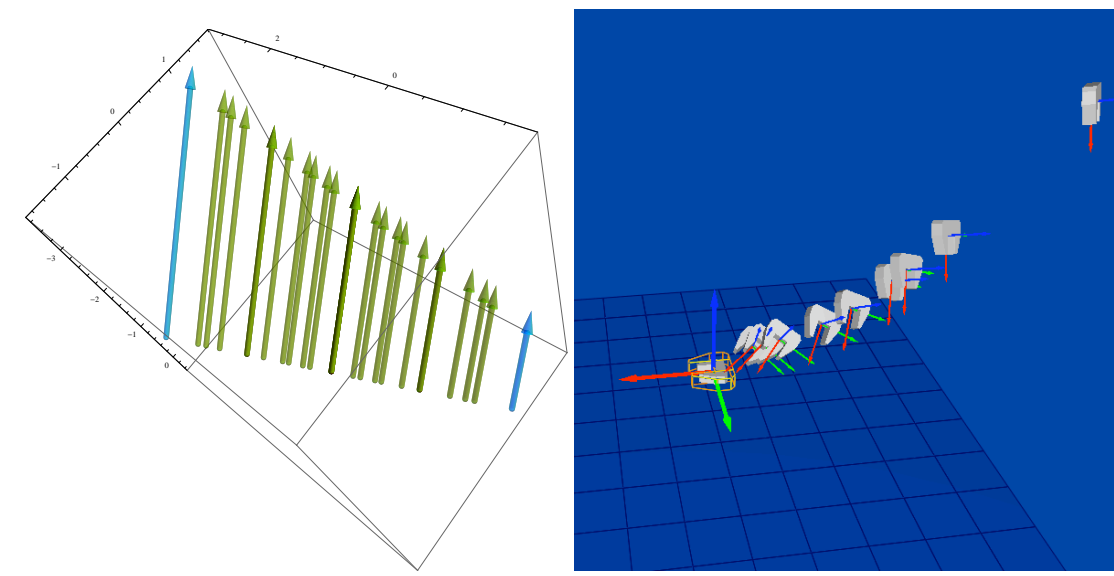

Fig. 2 Left, Screw system generated by $S_{12}$ and $S_{13}$ (shown in corners); right, corresponding absolute displacements.

Table 2 Joint axes for the RPRP linkage at the reference configuration

\begin{tabular}{|c|c|c|c|c|}
\hline Chain & Revolute joint $\mathrm{G}$ & Prismatic joint $\mathbf{h}$ & $\begin{array}{l}\text { Rotations } \\
\left(\theta_{12}, \theta_{13}\right)\end{array}$ & Slides $\left(d_{12}, d_{13}\right)$ \\
\hline $\mathrm{RP}$ & $\begin{array}{l}(-0.620,0.179,0.763)+ \\
\varepsilon(-0.436,-3.166,0.389)\end{array}$ & $(-0.087,0.893,0.442)$ & $(-264.5,-25.2)$ & $(5.30,-3.05)$ \\
\hline PR & $\begin{array}{l}(0.620,-0.179,-0.763)+ \\
\varepsilon(2.316,-1.826,2.310)\end{array}$ & $(-0.274,0.065,-0.959)$ & $(264.5,25.2)$ & $(-5.30,3.05)$ \\
\hline
\end{tabular}

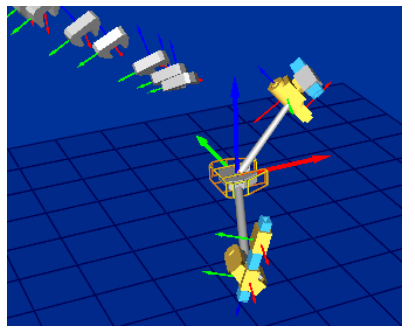

Fig. 3 The RPRP linkage reaching the first position

\section{Conclusions}

This papers presents the synthesis of an overconstrained closed linkage, the RPRP. The knowledge of the screw system that corresponds to the finite displacements of the linkage is used to ensure that the solutions of the synthesis of the RP and PR serial chains can be assembled to create a movable system. The counting of the maximum positions for the synthesis suffices to define the positions that generate the screw system. The synthesis yields a single RPRP linkage. 


\section{References}

1. J.E. Baker. On the motion geometry of the bennett linkage. In Proc. 8th Internat. Conf. on Engineering Computer Graphics and Descriptive Geometry, pages 433-437, Austin, Texas, USA, 1998.

2. D. Chablat and P. Wenger. Architecture optimization of a 3-dof parallel mechanism for machining applications, the orthoglide. IEEE Transactions on Robotics and Automation, 19(3):403-410, 2003.

3. G. Gogu. Structural Synthesis of Parallel Robots. Part 1: Methodology. Springer, first edition, 2007.

4. C. Huang. On the finite screw system of the third order associated with a revolute-revolute chain. ASME Journal of Mechanical Design, 116:875-883, 1994.

5. C. Huang. The cylindroid associated with finite motions of the bennett mechanism. In Proceedings of the ASME Design Engineering Technical Conferences, 1996, Irvine, CA, USA, 1996.

6. C. Huang. Linear property of the screw surface of the spatial rprp linkage. ASME Journal of Mechanical Design, 128:581-586, 2006.

7. T. Huang, M. Li, X.M. Zhao, J.P. Mei, D.G. Chetwynd, and S.J. Hu. Conceptual design and dimensional synthesis for a 3-dof module of the trivariant - a novel 5-dof reconfigurable hybrid robot. IEEE Transactions on Robotics, 21(3):449-456, 2005.

8. K.H. Hunt. Kinematic Geometry of Mechanisms. , Oxford University Press, 1978.

9. M.L. Husty, M. Pfurner, H.-P. Schrocker, and K. Brunnthaler. Algebraic methods in mechanism analysis and synthesis. Robotica, 25:661-675, 2007.

10. H.S. Kim and L.-W. Tsai. Design optimization of a cartesian parallel manipulator. ASME Journal of Mechanical Design, 125:43-51, 2003.

11. H.S. Kim and L.-W. Tsai. Kinematic synthesis of a spatial 3-rps parallel manipulator. ASME Journal of Mechanical Design, 125:92-97, 2003.

12. A. Kosinska, M. Galicki, and K. Kedzior. Design and optimization of parameters of delta-4 parallel manipulator for a given workspace. Journal of Robotic Systems, 20(9):539-548, 2003.

13. E. Lee and C. Mavroidis. Solving the geometric design problem of spatial 3r robot manipulators using polynomial homotopy continuation. ASME Journal of Mechanical Design, 124(4):652-661, 2002.

14. J.-P. Merlet. Optimal design of robots. In Proceedings of Robotics: Science and Systems, June, 2005, Cambridge, USA, 2005.

15. I.A. Parkin. A third conformation with the screw systems: Finite twist displacements of a directed line and point. Mechanism and Machine Theory, 27:177-188, 1992.

16. A. Perez and J. M. McCarthy. Dimensional synthesis of bennett linkages. ASME Journal of Mechanical Design, 125(1):98-104, 2003.

17. A. Perez and J. M. McCarthy. Dual quaternion synthesis of constrained robotic systems. ASME Journal of Mechanical Design, 126(3):425-435, 2004.

18. A. Perez Gracia and J. M. McCarthy. The kinematic synthesis of spatial serial chains using clifford algebra exponentials. Proceedings of the Institution of Mechanical Engineers, Part $C$, Journal of Mechanical Engineering Science, 220(7):953-968, 2006.

19. M. Pfurner and M.L. Husty. A method to determine the motion of overconstrained 6rmechanisms. In Proceedings of the 12th IFToMM World Congress, June 18-21, 2007, Besancon, France, 2007.

20. N.M. Rao and K.M. Rao. Dimensional synthesis of a 3-rps parallel manipulator for a prescribed range of motion of spherical joints. Mechanism and Machine Theory, 44:477-486, 2009.

21. E. Wolbrecht, H.-J. Su, A. Perez, and J.M. McCarthy. Geometric design of symmetric 3-rrs constrained parallel platforms. In ASME, editor, Proceedings of the 2004 ASME International Mechanical Engineering Congress and Exposition, November 13-19, 2004, Anaheim, California, USA, 2004.

22. D. Zlatanov, S. Agrawal, and C.L. Gosselin. Convex cones in screw spaces. Mechanism and Machine Theory, 40:710-727, 2005. 